\title{
Traumdarstellungen als Narratoid. Epistemische Sprünge bei der Wiedergabe von Träumen
}

\section{Die Traummitteilung als „unproblematisches Problem“}

Der sozialwissenschaftlichen Gattungsanalyse, die - angestoßen durch Arbeiten von Thomas Luckmann - in den 1980er Jahren ihren Ausgang nahm, liegt der Gedanke zugrunde, dass in jeder Gesellschaft verfestigte kommunikative Formen als kulturelle ,ready-mades‘ zur Verfügung stehen, weil sich diese Muster als Lösungen für wiederkehrende kommunikative Probleme herausgebildet und konventionalisiert haben. In Fortführung der sozialphänomenologischen Arbeiten von Alfred Schütz argumentiert Thomas Luckmann, dass die einzelnen Akteure bei ihren Handlungen durch gesellschaftlich vorgegebene Einrichtungen wie etwa die Sprache, kanonisierte Wissensbestände etc. von eigenständigen Sinnsetzungen und -findungen entlastet werden müssen. Dies führt zu der Überlegung, dass es derartige Entlastungsmechanismen auch für die Lösung kommunikativer Probleme gibt, da andernfalls die Akteure bei jeder kommunikativen Aufgabe immer wieder erneut singuläre Lösungen entwickeln müssten (Luckmann 1986: 202). Wiederkehrende kommunikative Probleme entstehen etwa in Situationen, in denen entschieden werden muss, wie man einer unbekannten Person begegnet, wie man ein Gespräch beginnt, wie man einen Konflikt löst, wie man Wissen übermittelt, wie man jemanden überzeugt, wie man mit der Verletzung einer Verhaltensregel umgeht, wie man eine kommunikative Begegnung beendet etc. Für rekurrente „Probleme“ dieser Art haben sich in Gestalt der kommunikativen Gattungen routinemäßige „Lösungen“ herausgebildet, die dafür sorgen, dass diese Probleme im Alltag als „unproblematische Probleme“ erfahren werden. ${ }^{1}$

Die Wiedergabe von Träumen, um die es in der folgenden Studie geht, ist im vorgenannten Sinn eines jener wiederkehrenden kommunikativen Probleme,

1 Von „unproblematischen Problemen“ in diesem Sinn sprechen Berger/Luckmann (1970: 27); für eine Übersicht über Konzeption, theoretische Begründung und empirische Vielfalt der Gattungsforschung vgl. Günthner/Knoblauch (1997). 
mit dem sich jeder, der beabsichtigt, einen Traum mitzuteilen, konfrontiert sieht. Träume sind ein ubiquitäres Phänomen und gleichzeitig eine vollkommen subjektive Erfahrung. Wer träumt, tut dies notwendigerweise allein und steht damit vor der Frage, wie dieses Ereignis aus dem inneren Erlebnisraum kommunikativ übermittelt werden kann. Diese Frage stellt sich allerdings nicht nur für die Darstellung eines Traums, denn der Traum der vergangenen Nacht ist nur eines von unendlich vielen Ereignissen, die aus der Vergangenheit durch Kommunikation in die Jetztzeit zurückgeholt und vergegenwärtigt werden können. Für diese Aufgabe hat sich die große Familie der narrativen Gattungen entwickelt, die dafür sorgen, dass sich Ereignisse, nachdem sie geschehen sind, nicht einfach in nichts auflösen, sondern aus dem Gedächtnis benannt, thematisiert, erzählt und auf andere Weise im Gespräch rekonstruiert werden können (vgl. auch Gülich i.d. B. und Kotthoff i.d. B.). Für Traumdarstellungen ist daher zu vermuten, dass sich als Lösung für das beharrliche „Problem“, wie die subjektive Erfahrung eines Traums intersubjektiv vermittelt werden kann, ein im Umfeld der narrativen Gattungen angesiedeltes kommunikatives Muster - eine Erzählform? - etabliert hat.

Bereits in der Alltagserfahrung zeigt sich jedoch, dass sich Träume gegen ein einfaches Erzählt-Werden sperren: Träume bestehen - nicht nur unmittelbar nach dem Erwachen - zumeist aus unzusammenhängenden Szenen, Ereignisfetzen und bizarren Einzelheiten. Die bisher vorliegenden Untersuchungen, die sich nicht dem Inhalt, sondern der kommunikativen Darstellung eines Traums widmen, zeigen denn auch, dass sich die Praktiken der Mitteilung von Träumen weder bruchlos unter das Schema des Erzählens subsumieren lassen noch eine eigene kommunikative Gattung konstituieren. Bergmann (2000: 56) beobachtet, dass sich Träume aufgrund ihrer episodisch-zersplitterten Qualität gegen ihre narrative Glättung sträuben und die Darstellung eines Traums fortwährend davon bedroht ist, , an den identitätsgefährdenden Implikationen, den Leistungsgrenzen der kommunikativen Form oder anderen Faktoren zu scheitern“; Boothe gelangt zu der Feststellung: „Die Traummitteilung ist keine Erzählung, sondern ein Narratogen. [...] Dem Traumbericht fehlt die motivierende Klammer. Der Darstellung fehlt die egozentrische Involviertheit, die für Alltagserzählungen charakteristisch ist“ (2008: 42); Hausendorf (2012) untersucht, wie es trotz der „prekären Erzählbarkeit“ von Träumen zu Traumerzählungen kommen kann; und Gülich/Hausendorf (2012) machen den Vorschlag, verschiedene Weisen der Traumdarstellung - die Traumerwähnung, die Traummitteilung, verschiedene narrative Weisen der Traumrekonstruktion - zu differenzieren. 


\section{Die epistemische Spezifität der Traumwahrnehmung}

Entlang dieser Untersuchungslinie soll im Folgenden der epistemische Status von Traumdarstellungen genauer analysiert werden. Träume sind subjektive Erfahrungen und können als solche mit anderen subjektiven Erfahrungen verglichen werden - Erfahrungen, die entweder als Wahrnehmungen über die fünf Sinne vermittelt werden, oder Erfahrungen, die außer- oder übersinnlicher Natur sind. In der Art und Weise, wie subjektive Erfahrungen dieser Art in der Alltagskommunikation dargestellt werden, zeigen sich im Vergleich zur Darstellung von Traumerfahrungen signifikante Unterschiede, die auf den besonderen epistemischen Status von Träumen verweisen.

- Gegenüber der subjektiven Abgeschlossenheit eines Traums besteht für die wache visuelle oder akustische Wahrnehmung eines Ereignisses in der Alltagswelt immer die Möglichkeit, dass das Ereignis auch von anderen Beobachtern oder Zuhörern bezeugt wurde und damit der Wahrheitsanspruch einer Darstellung zweifelhaft werden kann; zudem muss die Darstellung eines Ereignisses in der Alltagswelt Kausalzusammenhängen genügen und in ihrem Sinngehalt plausibel sein.

- Geruch, Geschmack und taktiler Sinn sind zwar ebenfalls subjektive Wahrnehmungsmodi, doch auch Geschmacksurteile unterliegen einer Plausibilitätsforderung und müssen einen nachvollziehbaren Zusammenhang zwischen dem Ausgangsstimulus und der Geschmackswahrnehmung herstellen (oder die Inkongruenz z. B. bei der Verkostung einer exotischen Speise als Merkwürdigkeit markieren).

- Schmerzempfindungen sind zwar „privater“ Natur, doch ihre Mitteilung wird erst dann verständlich, wenn der Schmerz auf eine plausible Schmerzursache zurückgeführt werden kann. Schmerzen ohne diagnostizierbare Ursache sind deshalb ein besonderer Fall für medizinische Spezialisten (oder andere „Heiler“), die dann nach ganz abwegig erscheinenden Ursachen forschen.

- Die Mitteilung religiöser oder spiritueller Erfahrungen, die für andere unzugänglich sind, trifft bei den Mitmenschen in hohem Maße auf Zweifel und ist, sofern sie nicht in einem gesellschaftlich akzeptierten Glaubenssystem lokalisiert sind, der Gefahr psychopathologischer Zuschreibungen ausgesetzt. ${ }^{2}$

2 Man könnte noch Fantasien, Tagträume, Wachträume oder ,luzide Träume‘ als subjektive Erfahrungen eigener Art hinzufügen. Diese subjektiven Erfahrungsmodi werden jedoch in der Regel 
In den hier genannten Wahrnehmungsmodi werden Darstellungen subjektiver Erfahrungen prinzipiell in einer Art „Realitäts-Check“ im Hinblick auf ihre Plausibilität, Sinnhaftigkeit und kausale Logik überprüft. Wenn diese Prüfung nicht bestanden wird, wenn also keine Korrespondenz zwischen einer Wahrnehmung und einem alltagsweltlichen Ereignis oder Sachverhalt hergestellt werden kann, ist die Wahrscheinlichkeit groß, dass die mitgeteilte Wahrnehmung als bloßer Irrtum, als Verzerrung, Halluzination oder Vortäuschung eingestuft wird.

Im Gegensatz zur Mitteilung anderer subjektiver Wahrnehmungen unterliegt die Darstellung eines Traums keiner empirischen Korrespondenzprüfung und Plausibilitätspflicht. Traumdarstellungen lassen sich prinzipiell nicht im Hinblick auf ihren Wahrheitsgehalt überprüfen und könnten theoretisch frei erfundene Phantasiegeschichten sein. Der in einer Traumschilderung enthaltene Geltungsanspruch, dass es sich dabei um einen real erlebten Traum und nicht um eine ausgedachte Geschichte handelt, kann von niemandem außer dem Träumer selbst validiert werden. Aufgrund der dem Traum gestatteten Lockerung logischer Selbstverständlichkeiten sind auch Stimmigkeit, Plausibilität oder Nicht-Widersprüchlichkeit keine Kriterien zur Beurteilung des Wahrheitsgehalts einer Traumdarstellung. Der Wahrhaftigkeitsanspruch einer Traumdarstellung muss deshalb als prekär gelten, was zu der Frage führt, wie Sprecher, die einen Traum wiedergeben, in der Rekonstruktion eines Traums diese epistemische Problematik berücksichtigen.

\section{Die narrative Eröffnung einer Traummitteilung}

Während Traumdarstellungen in der Alltagskommunikation eher selten sind, ${ }^{3}$ spielen Träume und ihre unzensierte Wiedergabe in der Psychoanalyse eine zentrale Rolle. Für die konversationsanalytische Untersuchung von Traumdarstellungen sind psychoanalytische Therapiesitzungen daher eine reichhaltige Quelle. ${ }^{4}$

in der Nachbarschaft von Träumen verortet, auch wenn ihr epistemischer Status noch weitgehend ungeklärt ist, vgl. aber Bernhard (2011).

3 Über die vielfältigen Gründe für diese Beobachtung vgl. Bergmann (2000: 50-54). Die bislang ausführlichste Untersuchung über das konversationelle Traumerzählen von Hanke (2001) stützt sich auf Datenmaterial aus einer ,Traumgruppe‘, bei der sich mehrere Personen in unregelmäßigen Abständen mit dem spezifischen Zweck trafen, sich ihre Träume zu erzählen. Weil Träume oft im Kontext intimer Gemeinschaften, geteilt‘ werden, schlägt Tedlock (1991) vor, dass Feldforscher Träume nicht in Interviews erfragen, sondern durch das Erzählen eigener Träume elizitieren sollen.

4 Zum Verhältnis von Psychoanalyse und Konversationsanalyse prinzipiell vgl. Peräkylä (2008). 
Die folgenden Analysen basieren auf Traumdarstellungen aus einer psychoanalytischen Therapie, die unter der Bezeichnung „Amalie X“ in der „Ulmer Textbank“ dokumentiert ist und als „specimen case“ bereits Gegenstand zahlreicher Untersuchungen war (vgl. Kächele et al. 2006). Der Fall enthält die Tonbandaufzeichnungen und Transkripte einer mehrjährigen Psychoanalyse mit Amalie X, einer 30-jährigen Frau, die in Folge ihres Hirsutismus (männlicher Haarwuchs am ganzen Körper) an stark eingeschränktem Selbstwertgefühl und depressiven Stimmungen litt. Dokumentiert ist die gesamte psychoanalytische Therapie mit über 500 Sitzungen, in deren Verlauf Amalie X nahezu 100 Träume mitteilte. ${ }^{5}$ Das folgende Segment stammt aus der 104. Therapiesitzung.

\section{Beispiel 1: [Amalie 104:12]}

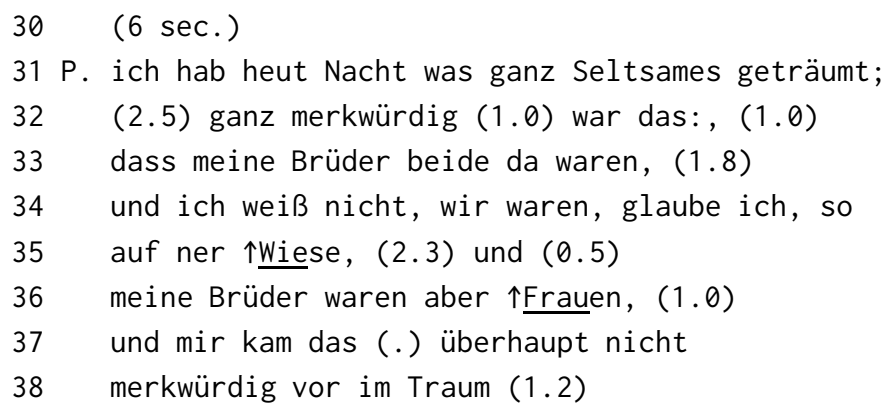

Etwa zehn Minuten nach Sitzungsbeginn und nach einer Pause von mehreren Sekunden beginnt Amalie X damit, ohne thematische Anbindung an vorausgegangene Äußerungen einen Traum wiederzugeben. Die Darstellung zeichnet sich dadurch aus, dass sie nach dem Muster einer narrativen Exposition erfolgt.

Die Erzählerin führt zunächst sich selbst als diejenige ein, die eine Erfahrung gemacht hat (,ich hab“), und sie spezifiziert sowohl den Zeitpunkt der Erfahrung (,heut Nacht“) als auch den Erfahrungsmodus („geträumt“). Die Markierung der nachfolgenden Darstellung als „Traum“ ist ein charakteristisches Merkmal aller Traummitteilungen; sie dient als Rahmung für die anschließende Rekonstruktion, die sich auf einen geschlossenen Sinnbereich, eine eigene umgrenzte „Sinnprovinz“ (Schütz 1971: 266) bezieht, in der die Fraglosigkeiten der Alltagswelt verschwunden sind und die Gesetze der Logik ihre Geltung verloren haben.

5 Die in der Ulmer Datenbank vorliegenden Transkripte des Falles Amalie X sind verhältnismäBig grob, sie wurden auf der Grundlage der verfügbaren Tonbandaufzeichnungen von den Autoren auf der Grundlage der von Gail Jefferson entwickelten Konventionen re-transkribiert, die weitgehend den Regelns des GAT-Minimaltranskripts entsprechen; vgl. Selting et al. (2009: 359). Ein Inventar aller Träume im Fall Amalie X findet sich als Anhang zu dem Text von Boothe (2008). 
Eine weitere Komponente der narrativen Exposition ist die vorgreifende Evaluation der nachfolgenden Darstellung. Die Wiedergabe des Traumes antizipierend, charakterisiert die Sprecherin den Traum als „was ganz Seltsames“, und in einer zweiten Einschätzung fügt sie hinzu: „(2.5) ganz merkwürdig (1.0) war das:, (1.0)“. In seiner Analyse solcher „story prefaces“ hat Harvey Sacks gezeigt, dass Charakterisierungen dieser Art nicht nur als Mittel der Erzeugung von Spannung und Interesse bei den Zuhörern dienen, sondern auch eine interaktionsorganisatorische Funktion haben: Sie garantieren der Sprecherin das Rederecht für die Erzählung einer Geschichte, eines Witzes etc., für die sie üblicherweise mehr als einen Redezug benötigt, und: „the characterizing adjective for the story is used by the hearer of the story to produce their remark on recognition that the story is over“ (Sacks: 1992 [1968]: 10). Die vorgreifende Charakterisierung bringt die ZuhörerInnen also dazu, aufmerksam zuzuhören, um die Pointe einer Geschichte nicht zu verpassen.

In der einleitenden Rahmung einer Geschichte werden typischerweise die Protagonisten der Erzählung benannt oder vorgestellt - im vorliegenden Fall die Sprecherin selbst und ihre „Brüder“ („wir“) - und es werden Hinweise zur räumlichen Lokalisierung eines Ereignisses gegeben (,wir waren, glaube ich, so auf ner 个Wiese,“).

Bis hierhin folgt Amalies Mitteilung eines Traums der Vorbereitung einer Geschichte, sie entspricht ganz dem Schema der Initiierung einer Erzählung, die im Alltag beginnen könnte mit: ,gestern ist mir im Zug was Unglaubliches passiert ...“. Allerdings lassen sich einige Elemente identifizieren, die sich nicht so ohne Weiteres in das gängige Muster der narrativen Exposition fügen wollen. Da ist zum einen die frühe Auflösung dessen, was in der Rahmung vorgreifend als „seltsam“ bzw. „merkwürdig“ charakterisiert wurde. Es scheint fast so, als würde durch die Aussage „meine Brüder waren aber 个Frauen“ (Z. 36) die Pointe bereits offengelegt, noch ehe die Geschichte erzählt wurde. Dazu kommt, dass die Geschichte aufgrund eines fehlenden Handlungsmoments eigentlich gar nicht als Erzählung gelten kann, sondern eher als eine - überraschende - Sachverhaltsdarstellung bezeichnet werden muss. Auffällig ist zudem, dass die Sprecherin verschiedene epistemische Markierungen vornimmt, die sowohl ihr Wissen über den Traum (,und ich weiß nicht, “ Z. 34) als auch ihre Charakterisierung des Traums als „merkwürdig“ relativiert (,(1.0) und mir kam das (.) überhaupt nicht merkwürdig vor im Traum (1.2)“ Z. 37/38). Diese Aspekte sollen im Weiteren genauer untersucht werden. 


\section{Limitiertes Traumwissen}

Immer wieder platziert die Sprecherin in \#1 während der Darstellung eines Traums Unsicherheitsmarkierungen, indem sie explizit ihr Nichtwissen formuliert („ich weiß nicht“ Z. 34) oder auf andere Weise zum Ausdruck bringt, dass ihr Wissen begrenzt oder ihre Erinnerung ungenau ist („glaube ich,“ Z. 34). Wenige Sekunden nach Segment \#1 finden sich die folgenden Passagen:

\section{Beispiel 2: [Amalie 104:12]}

$49 \mathrm{P}$. und zwar hatten die beide so so Sommerkleider an mit

50 mit ziemlichem Dekolleté, .hhh und dann (1.0) 1- (0.2)

51 lag ich in der Mitte? mein ältester Bruder rechts $\uparrow$ und

52 links (.) (lag) (.) [Name], und, (1.5) und dann weiß

53 ich noch dass ich gesagt hab, @ach was, die haben ja

54 ein viel schöneres Dekolleté als ich.@ ja, immer

55 meine Brüder, (.) die haben alles besser.

$56 \mathrm{~T} . \mathrm{mmh}$.

$57(0.8)$

\section{Beispiel 3: [Amalie 104:12]}

$78 \mathrm{P}$. die kontrollieren mich@. Und dann weiß ich nicht mehr<

79 (1.0) hat dann jemand gesagt, ich weiß nicht, ob das

80 mein Bruder war, na ja, ...

Formulierungen wie „dann weiß ich noch“ (Z. 52/53) oder „dann weiß ich nicht mehr“ (Z. 78) geben zu erkennen, dass die Sprecherin ihre Traumdarstellung auf eine Erinnerung gründet, die insofern dynamischer Art ist, als sie schwächer werden und bestimmte Inhalte verlieren oder durch angestrengtes Überlegen wieder zurückholen kann. Die Erinnerung hat keine feste Gestalt, sie ist nicht entweder da oder nicht da, vielmehr kann sie vage oder präzis, frisch oder halb vergessen sein und durch entsprechende Anstrengungen dem Gegenstand der Erinnerung schrittweise näherkommen. Wie sehr das Erinnern eines Traumes gekennzeichnet ist durch das Bemühen der Sprecherin um die genaue Darstellung einer Traumerfahrung, zeigt die folgende Episode aus der gleichen Therapiesitzung mit Amalie:

\section{Beispiel 4: [Amalie 104:14]}

194 P. . . . erst (.) nachher vertauscht, als [Name] wegfuhr 195 T. mhm.

$196 \mathrm{P}$. oder wegging da dur[ch diesen (-) durch diese Allee 197 T.

$[\mathrm{Mm}$, 
198 P. oder durch diesen Wald, nein, es war so mehr ein 199 Park.

In diesem Ausschnitt ist zu sehen, wie die Sprecherin um eine genaue Darstellung ihrer Traumerfahrung ringt, mehrfach berichtigt sie Beschreibungen oder nimmt zunächst gewählte Deskriptoren zurück und ersetzt sie durch andere. Nachdem sie im Zug ihrer Traumschilderung zunächst die Tätigkeitsbeschreibung „wegfuhr" durch „wegging“ (Z. 194/196) korrigiert, kommt es zu einer Substitutionsreihe, in deren Verlauf eine Handlungsumgebung zuerst als „Allee“, dann als „Wald“ und danach als „Park“ (Z. 196/198/199) bezeichnet wird. Was dieses Bemühen um eine genaue sprachliche Repräsentation des Traumereignisses bemerkenswert macht, ist der Sachverhalt, dass der Zuhörer keinerlei Möglichkeit hat, die Angemessenheit einer dieser Beschreibungen zu beurteilen. Für ihn sind alle drei Beschreibungen gleichermaßen möglich, denn wie könnte er, dem der Traum selbst völlig verschlossen ist, entscheiden, ob es sich bei der Umgebung des Traumgeschehens um eine Allee, einen Wald oder einen Park gehandelt hat zumal ja auch „Plausibilität“ als Beurteilungskriterium ausscheidet?

Die Unsicherheitsmarkierungen und Selbstkorrekturen in der Wiedergabe eines Traums beziehen sich ganz offensichtlich nicht auf die Sprecher-Zuhörer-Relation, sondern auf die Relation Sprecher-Traumerfahrung. Damit kommt diesen Reparaturen und Reformulierungen, die ja bei der Rekapitulation einer Erinnerung prinzipiell nichts Ungewöhnliches sind, eine besondere epistemische Funktion in der Wiedergabe eines Traumes zu. Wenn eine Sprecherin in der Darstellung ihrer Traumerfahrung ihre Beschreibungen fortwährend nachjustiert, durch Korrekturen anpasst oder auf lückenhaftes Wissen verweist, wird das so Dargestellte zu einem unabhängig von der Sprecherin existierenden Objekt, das in der Darstellung - und in der Erinnerung - immer nur näherungsweise erfasst werden kann. Nicht die Bezeichnung, sondern die Korrektur erschafft gewissermaßen das Objekt, welches sie korrigiert. Der mehrfache Verweis auf das limitierte Wissen über den Traum und die wiederholte Kalibrierung der Beschreibung machen aus dem Traum ein externes Objekt, womit das epistemische Problem, dass der Traum eine unhintergehbar subjektive Erfahrung ist, gelöst wird. Mit der Objektivierung des Traums ergibt sich für die Sprecherin die Möglichkeit, eine Differenzierung zwischen dem Traum als einem tatsächlichen Ereignis und der sprachlichen Darstellung des Traums vorzunehmen. Dabei spaltet sie sich auf in jemanden, die einen Traum hatte, und jemanden, die den Traum als ein Ereignis beobachtet, jedoch nur ein eingeschränktes - und damit fehlerhaftes und korrekturbedürftiges - Erinnerungswissen über den Traum besitzt. 


\section{Auktoriale Darstellungsperspektive}

Zwar erscheint der Traum durch diese Strategie zur Objektivierung wie ein Ereignis, das aus der Distanz beobachtet wurde, dennoch ist der Traum als eine Erfahrung, die das träumende Subjekt allein hervorgebracht hat, in den Traumschilderungen nicht verschwunden. Das zeigt sich darin, dass in den Traumdarstellungen immer wieder Passagen enthalten sind, in denen sich die Sprecherin als allwissend erweist, in die Zukunft blicken kann oder Motive und Absichten der Akteure kennt, so etwa in dem folgenden Ausschnitt:

\section{Beispiel 5: [Amalie \#152:03]}

18 P. ich hab heut Nacht geträumt, heut morgen, (da hat grad)

19 der Wecker gschellt. ich sei ermordet worden vom Dolch.

$20 \mathrm{~T} . \mathrm{mhm}$.

$21 \mathrm{P}$. und zwar war's aber- (0.5) wie im Film (1.2) und ich

22 musste ganz lang liegen auf dem Bauch, (.) und hatte

23 den Dolch im Rücken und, dann kamen ganz viele Leute,

24 (3.0) und, ich weiß nicht mehr genau, die Hände ganz

25 ruhig halten, irgendwie (wie tot)

$26 \mathrm{~T}$. mhm.

$27 \mathrm{P}$. mir war's sehr peinlich dass der Rock so $h(h)$ och

28 raufgerutscht war hinten

29 T. mhm.

30 P. und dann kam (.) ein Kollege, ganz deutlich sichtbar 31 aus $X Y$, das war meine allererste Stelle, und der hat

32 dann den Dolch aus dem Rücken gezogen und mitgenommen.

33 und ich weiß es war wie ein Souvenir dann. und dann kam

34 ein junges Paar, (1.5) ich weiß nur dass er ein Neger

35 war. und die haben mir dann die Haare abgeschnitten und

36 wollten daraus, tatsächlich ne Perücke glaub ich machen.

37 und das fand ich ganz schrecklich. einfach alles runter

38 und die haben dann auch angefangen zu schneiden. (.)

39 und, ich bin dann aufgestanden, (.) und $b(h)$ in zum Friseur.

40 und (.) da hat d' $n($.$) der (Wecker)( ) geschellt ( )$

41 und bin aufgewacht.

Einerseits sind in diese Traumschilderung die bereits beschriebenen Unsicherheitsmarkierungen eingeflochten (Z. 24/25: „ich weiß nicht mehr genau“, „irgendwie“), andererseits finden sich in der Darstellung zahlreiche Informationen und Details, die nur ein allwissender Beobachter kennen kann, der fähig ist, in die 
Köpfe der Akteure zu blicken. So bemerkt P. etwa zur Mitnahme des Dolches: ,und ich weiß es war wie ein Souvenir dann" (Z. 33), und kurze Zeit später ist ihr die Absicht eines jungen Paares bekannt, aus ihren abgeschnittenen Haaren eine Perücke zu machen. Dabei ist ihre Formulierung -

36 wollten daraus, tatsächlich ne Perücke glaub ich machen.

- insofern signifikant, als sich in ihr die auktoriale Perspektive der Sprecherin („wollten daraus tatsächlich ne Perücke [...] machen“) vereint mit einer nichtauktorialen Perspektive, in der die Sprecherin nur ein eingeschränktes Wissen über das von ihr geschilderte Geschehen hat („glaub ich“). Dieses Zugleich von allwissender und subjektiv beschränkter, von auktorialer und nicht-auktorialer Darstellungsweise ist ein typisches Merkmal von Traumdarstellungen. In diesem Zugleich kommt zum Ausdruck, dass der Traumdarsteller immer beides ist: Produzent wie Rezipient des Traums, Schöpfer wie Opfer des Traums, Sprecher des aktiven „ich träumte“, wie des passiven „mir träumte“. Wer einen Traum schildert, besitzt hierfür eine epistemische Autorität, da er ganz allein Urheber und Autor des Traumes ist; doch nur dadurch, dass in der Schilderung eines Traums Grenzen und Einschränkungen dieser epistemischen Autorität deutlich werden, wird der Traum zu einem objektiven Ereignis und die Traumdarstellung wahrhaftig.

Im Übrigen ist es dieses Zugleich von auktorialer und nicht-auktorialer Perspektive, das den Traum zu einem für die Psychotherapie so sehr geeigneten Objekt macht. In dieser Dualität versteckt sich ja die Frage, inwiefern der Träumer bzw. der Traumdarsteller bereit ist, das, was er berichtet, als Teil seiner selbst anzuerkennen, und für das, was er tut, Verantwortung zu übernehmen. Unabhängig von den jeweiligen Trauminhalten, auf die sich die verschiedenen psychotherapeutischen Lehren konzentrieren, ist also der Traum aufgrund seines besonderen epistemischen Charakters ein höchst passendes Vehikel psychotherapeutischer Kommunikation.

\section{Interpretationen ex post und in situ}

Die Gleichzeitigkeit von auktorialer und nicht-auktorialer Perspektive ist nicht die einzige epistemische Verwerfung, die in Traumdarstellungen beobachtet werden kann. In dem in \#1 wiedergegebenen Ausschnitt -

Beispiel 6: Beispiel 1: [Amalie 104:12]

$31 \mathrm{P}$. ich hab heut Nacht was ganz Seltsames geträumt;

32

(2.5) ganz merkwürdig (1.0) war das:,

$(1.0)$ 
dass meine Brüder beide da waren, (1.8)

34 und ich weiß nicht, wir waren, glaube ich, so

35 auf 'ner $\uparrow$ Wiese, (2.3) und (0.5)

36 meine Brüder waren aber $\uparrow$ Frauen, (1.0)

37 und mir kam das (.) überhaupt nicht

38 merkwürdig vor im Traum (1.2)

- spricht Amalie in der Ankündigung ihres Traums zunächst davon, dass sie „was ganz Seltsames geträumt“ habe. Dabei legt vor allem der paraphrasierende Zusatz „ganz merkwürdig (1.0) war das:,“ (Z.31) durch die Verwendung der Vergangenheitsform die Interpretation nahe, dass das Ereignis, dessen Erzählung die Sprecherin ankündigt, in der Traumerfahrung selbst „seltsam“ war. Jedoch dementiert die Sprecherin diese Interpretation einige Sekunden später. Im Hinblick auf den Sachverhalt, dass ihre Brüder im Traum Frauen waren, stellt sie fest: „mir kam das (.) überhaupt nicht merkwürdig vor im Traum (1.2)“. In diesem Fall differenziert die Sprecherin selbst zwischen der Erfahrungsebene des Traums und der Wahrnehmungs- und Beschreibungsebene der Traumrekonstruktion. In anderen Fällen ist es nicht so ohne Weiteres möglich, die Perspektive der Traumerfahrung und die Perspektive der Traumdarstellung ex post auseinanderzuhalten, so etwa in \#5, in dem sich folgende Beschreibung findet:

\section{Beispiel 7: Beispiel 5: [Amalie \#152:03] (vereinfacht)}

18 P. ich hab heut Nacht geträumt, heut morgen, (da hat grad)

19 der Wecker gschellt. ich sei ermordet worden vom Dolch.

22 ich musste ganz lang liegen auf dem Bauch, (.) und hatte

23 den Dolch im Rücken

27 mir war's sehr peinlich dass der Rock so $h(h) o c h$

28 raufgerutscht war hinten

In Amalies Äußerung „mir war's sehr peinlich dass... “ bezieht sich das „mir“ auf die Person der Ermordeten, d. h. das Peinliche des hochgerutschten Rocks wird in den Erfahrungsraum des Traums verlegt. Gleichzeitig öffnet sich jedoch auch in diesem Fall die Möglichkeit, dass es sich hierbei um eine ex-post-Charakterisierung handelt, denn wie die eingestreuten Lachpartikeln in der Beschreibung des Vorfalls - „so h(h)och raufgerutscht“ - erkennen lassen, ist es der Sprecherin im Hier und Jetzt der Erzählung peinlich, diesen Vorfall - oder ihre Peinlichkeit wegen des Vorfalls - zu beschreiben. Im Sinn Bachtins (1979: 195) könnte man hier von einer „hybriden Konstruktion“ sprechen, bei der sich zwei Horizonte kreuzen: die abgebildete Emotion (hier: die Peinlichkeit im Traum) und die abbildende Emotion (hier: die Peinlichkeit bei der Wiedergabe des Traums). Die re-präsentative Stimme der Darstellung eines vergangenen Traums wird überlagert von 
einer performativen Stimme, die in einer Art „re-enactment“ das dargestellte Gefühl präsentiert. ${ }^{6}$ Durch diese Konfundierung geraten Realitätsebenen durcheinander, und es entsteht eine epistemische Diffusität, bei der die Verwerfungslinien nicht klar auszumachen sind.

\section{Die Gestaltlosigkeit der Träume}

Eine weitere epistemische Eigenart von Traumdarstellungen ist bereits oben erwähnt und sehr früh in der Traumforschung beschrieben worden (vgl. Strümpell 1874: 82): Träume zerfallen zumeist in einzelne Szenen, die zusammenhanglos und gestaltlos aufeinanderfolgen, zwar einzeln für sich ein narratives Moment besitzen mögen, doch sich nicht zu einer Geschichte zusammenfügen wollen. In dem bereits zitierten Segment \#05 zeigt sich dieses Merkmal ganz deutlich daran, dass die Wiedergabe des Traums mit der Unterbrechung durch den Wecker endet:

\section{Beispiel 8: Beispiel 5: [Amalie \#152:03]}

$30 \mathrm{P}$ : und dann kam (.) ein Kollege, ganz deutlich sichtbar

31 aus $X Y$, das war meine allererste Stelle, und der hat

32 dann den Dolch aus dem Rücken gezogen und mitgenommen.

33 und ich weiß es war wie ein Souvenir dann. und dann kam

34 ein junges Paar, (1.5) ich weiß nur dass er ein Neger

35 war. und die haben mir dann die Haare abgeschnitten und

36 wollten daraus, tatsächlich ne Perücke glaub ich machen.

37 und das fand ich ganz schrecklich. einfach alles runter

38 und die haben dann auch angefangen zu schneiden. (.)

39 und, ich bin dann aufgestanden, (.) und $b(h)$ in zum Friseur.

40 und (.) da hat $d$ ' $n$ (.) der (Wecker)( ) geschellt (

41 und bin aufgewacht.

Wie dieser Ausschnitt ebenfalls deutlich macht, werden in der Traumrekonstruktion Einzelheiten genannt (hier etwa: das junge Paar, der Neger), die in keinerlei sinnvollem Zusammenhang stehen, oder Informationen eingefügt (hier: der Kol-

6 Günthner (1997: 115) hat anknüpfend an Bachtin im Detail gezeigt, wie kunstfertig prosodische Gestaltungsmittel eingesetzt werden können, um innerhalb einer Äußerung verschiedene ,Stimmen' gleichzeitig sprechen zu lassen. 
lege aus XY, Amalies erste Arbeitsstelle), deren Relevanz im Dunkeln bleibt. Im folgenden Ausschnitt ist zwar ansatzweise eine Erzählung zu erkennen -

\section{Beispiel 9: [Amalie \#035:06]}

39 (33.0)

$40 \mathrm{P}$. war heute nacht (1.0) auch so komisch ich hatte

41 geträumt meine Großmutter sei ermordet worden und mhh

42 (0.2) .hhh und dann: (0.5) packten alle die Koffer=

43 =zuerst standen sie um diese (.) Leiche rum und (0.6)

44 überlegten wer das getan hätte und, (1.2) und (2.0)

45 plötzlich (1.0) hatte ich so $\mathrm{n}$ schrecklichen Verdacht

46 (.) 's sei unsere (1.2) damalige Putzfrau gewesen

47 (0.4) die in Wirklichkeit ne ganz (0.6) reizende

48 Frau war un[d,

49 T. $\quad[\mathrm{hm}[$ : .

$50 \mathrm{P}$. $\quad[$ Mh .hh und dann packte alles $(0.6)$ weiß

51 nicht wer das war Fa个milie oder .hh (0.2) irgendjemand

$52(0.4)$ weiß es nicht (0.2) kannte die Leute nicht genau.

53 (0.8) und die packten dann alle (0.5) zusammen

54 die Koffer und ( 0.4$)$ ich sollte mich da beeilen und

55 (1.3) \wurd ich glaub ich mitgenommen ( ).

- doch auch in diesem Fall ist die narrative Logik des dargestellten Ereignisses eher eine projektive Leistung des Zuhörers als eine kommunikative Leistung der Sprecherin. Stattdessen wird hier wie in \#05 die Darstellung durch zwei Stilmerkmale bestimmt: Einzelne Vorgänge werden verständnislos nach Art einer behavioristischen Verhaltensbeschreibung registriert, und es kommt zu einer „und dann... und dann“-Reihung von Ereignissen, die in keinem erkennbaren oder allenfalls lockeren Sinnzusammenhang stehen. Die Gestaltlosigkeit der Träume und der fehlende Zusammenhang einzelner Traumbestandteile werden seit längerer Zeit bereits als einer der Gründe genannt, weshalb Träume so leicht vergessen werden (vgl. etwa Draaisma 2012).

\section{Fazit}

Wie ein Traum aus dem abgeschlossenen subjektiven Erfahrungskosmos herausgeholt und intersubjektiv vermittelt werden kann, ist ein prinzipielles kommunikatives „Problem“, das bei jeder Wiedergabe eines Traums gelöst werden muss und für dessen Lösung daher ein Muster - eine kommunikative Gattung - zu er- 
warten ist. Entgegen dieser Erwartung hat die Analyse von Traumdarstellungen aus psychoanalytischen Sitzungen kein klares Bild ergeben. Zwar wird die Wiedergabe eines Traums häufig durch eine narrative Exposition (vgl. auch Knoblauch i. d. B.) eingeleitet, und in der Rekonstruktion des Traums selbst finden sich zumeist erzählerische Episoden. Doch in ihrer Gesamtheit folgt die Darstellung eines Traums keiner narrativen Logik, es kommt zu einer Aneinanderreihung von Szenen, Erinnerungsfragmenten und Ereignissplittern, die in der Regel ohne Gestaltschließung endet. Deshalb kann eigentlich nur in seltenen Fällen von einer „Traumerzählung“ gesprochen werden, und dort, wo ein Traum ganz im Format einer Erzählung präsentiert wird, liegt der Verdacht nahe, dass dies das Ergebnis einer nachträglichen Narrativierung ist.

Damit stellt sich die Frage, ob die Wiedergabe eines Traums überhaupt einem Muster folgt, das gattungsartig verfestigter Natur ist. Zu überlegen wäre etwa, ob Traumdarstellungen eine Art Gattungshybrid bilden, das sich aus Teilkomponenten verschiedener Gattungen zusammensetzt. ${ }^{7}$ Doch die empirische Analyse von Traumdarstellungen hat nicht erkennen lassen, mit welcher anderen Gattung sich das narrative Format vermischt haben könnte. Näher liegt da schon, Traumdarstellungen als eine Art derivative Gattung zu betrachten, die sich aus der Ursprungsgattung der Erzählung entwickelt hat. Der „kommunikative Haushalt einer Gesellschaft“ (Luckmann 1986: 206), deren Kernbereich die kommunikativen Gattungen bilden, ist ja keine statische Größe, vielmehr sorgen gesellschaftliche und technische Entwicklungen fortwährend für eine Evolution der aktuell geltenden Kommunikationsformen. ${ }^{8}$ Das führt dann nicht unbedingt zu einer Ablösung, sondern zu einem Nebeneinander benachbarter oder entfernt verwandter Gattungen, die in einem derivativen Verhältnis zueinander stehen - so z. B. die beiden Gattungen der Begrüßung und des Grüße-Ausrichtens (Bergmann 1994).

Die empirischen Analysen lassen nun allerdings den Schluss, dass Darstellungen von Träumen eine abgeleitete narrative Untergattung bilden, nicht zu. Traumdarstellungen haben zweifellos einen narrativen Grundtenor, doch die typische Gestaltlosigkeit von Träumen, die epistemischen Sprünge in der Darstellung zwischen auktorialer und nicht-auktorialer Perspektive sowie die Verquickung von ex post- und in situ-Interpretationen konterkarieren und schwächen die nar-

\footnotetext{
7 Zwar ist seit einigen Jahrzehnten in allen Medien und Kunstformen eine Tendenz zur Sprengung von Gattungsgrenzen und zur Gattungshybridisierung zu beobachten (vgl. Zymner 2010: 52; 255), doch auch in der Alltagswelt haben sich solche Gattungsmischungen entwickelt, vgl. etwa die Studie von Marjorie Goodwin (1980) zum ,He said, she said'-Format, das sich durch eine Hybridisierung von Klatsch und Streit auszeichnet.

8 Für zwei historische Arbeiten, die die historische Transformation kommunikativer Gattungen eindrücklich nachzeichnen, vgl. Dinges (1993) und Zakharine (2005).
} 
rative Darstellungslogik in einem solchen Maß, dass das Endresultat in vielen Fällen nur mehr wenig mit dem Gattungsschema einer Erzählung zu tun hat. Immer wieder scheitert die Durchführung einer Traumerzählung an der NichtErzählbarkeit ihres Gegenstands. Eine ganz ähnliche Beobachtung machte vor einigen Jahren Jefferson (1988) in ihrer Analyse von „trouble-talk sequences“. Sie stellte fest, dass in Episoden, in denen die GesprächsteilnehmerInnen über Probleme, Ärgernisse und anderes Ungemach sprechen, eine begrenzte Anzahl von Komponenten zum Einsatz kommt (u.a. announcement, affiliation, optimistic projection), aus denen sich eine wohlgeordnete „trouble-telling sequence“ konstruieren ließ. Allerdings beobachtete sie auch, dass sich in ihrem Material kein einziges Beispiel fand, in dem die so konstruierte Sequenz tatsächlich vollständig realisiert wurde. Jefferson erklärte dieses Paradoxon einer „sequence that wasn't there“ (1988: 418) damit, dass Gespräche über Alltagsärgernisse zwar virtuell dem von ihr identifizierten sequenziellen Format folgen, dieses Format jedoch im Fortgang des Gesprächs durch lokale und interaktive Kontingenzen - z. B. durch die Kontamination mit anderen Handlungsprojekten - immer wieder verletzt und durchbrochen wird. Das Scheitern einer Traumdarstellung als Erzählung ist in diesem Sinn das Scheitern eines Formats in seiner Realisierung. Doch im Unterschied zu „trouble-talk sequences“ scheitern Traumerzählungen nicht an lokalen Kontingenzen, sondern an der Widerständigkeit ihres Objekts. Gattungsterminologisch wird man dem labilen Charakter von Traumdarstellungen am ehesten dadurch gerecht, dass man sie als Narratoid versteht, bei dem das angekündigte Projekt einer Erzählung (,ich hab heut Nacht was ganz Seltsames geträumt“) in seinem Vollzug immer nur bruchstückhaft realisiert werden kann.

\section{Literatur}

Bachtin, Michail M. (1979): Das Wort im Roman. In: Grübel, Rainer und Michail M. Bachtin (Hrsg.), Die Ästhetik des Wortes, S. 154-300. Frankfurt a. M.: Suhrkamp.

Berger, Peter L. und Thomas Luckmann (1970): Die gesellschaftliche Konstruktion der Wirklichkeit - Eine Theorie der Wissenssoziologie. Frankfurt a. M.: S. Fischer.

Bergmann, Jörg (1994): Kleine Lebenszeichen. Über Form, Funktion und Aktualität von Grußbotschaften im Alltag. In: Sprondel, Walter M. (Hrsg.), Die Objektivität der Ordnungen und ihre kommunikative Konstruktion. Für Thomas Luckmann, S. 192-225. Frankfurt a. M.: Suhrkamp.

Bergmann, Jörg (2000): Traumkonversation. In: Boothe, Brigitte (Hrsg.), Der Traum - 100 Jahre nach Freuds Traumdeutung, S. 41-57. Zürich: vdf, Hochschulverlag an der ETH.

Bernhard, Peter (2011): Epistemologische Implikationen luzider Träume. In: Internationale Zeitschrift für Philosophie und Psychosomatik, 1:1-8. 
Boothe, Brigitte (2008): Initialträume und Finalträume im systematischen Vergleich. Eine Fallformulierung im Spiegel des Traumnarrativs. In: Psychotherapie und Sozialwissenschaft, 10:41-72.

Dinges, Martin (1993): Ehrenhändel als „Kommunikative Gattungen“ - Kultureller Wandel und Volkskulturbegriff. In: Archiv für Kulturgeschichte, 75(2):359-393.

Draaisma, Douwe (2012): Das Buch des Vergessens - Warum Träume so schnell verloren gehen und sich unsere Erinnerungen ständig verändern. Berlin: Kiepenheuer \& Witsch.

Goodwin, Marjorie (1980): He-said-she-said - Formal cultural procedures for the construction of a gossip dispute activity. In: American Ethnologist, 7(4):674-695.

Gülich, Elisabeth und Heiko Hausendorf (2012): Träume im Gespräch. Linguistische Überlegungen zur Erzählbarkeit von Träumen. In: Kern, Friederike, Miriam Morek und Sören Ohlhus (Hrsg.), Erzählen als Form - Formen des Erzählens, S. 13-47. Berlin: de Gruyter.

Gülich, Elisabeth (in Zusammenarbeit mit Martin Schöndienst) (i. d. B.): „Ich dachte, Sie stellen Fragen.“ Irritationen und Aushandlungsprozesse im Zusammenhang mit Gattungserwartungen. In: Weidner, Beate et al. (Hrsg.), Verfestigungen in der Interaktion. Konstruktionen, sequenzielle Muster, kommunikative Gattungen. Berlin: de Gruyter.

Günthner, Susanne (1997): Stilisierungsverfahren in der Redewiedergabe - Die „Überlagerung von Stimmen“ als Mittel der moralischen Verurteilung in Vorwurfsrekonstruktionen. In: Selting, Margret und Barbara Sandig (Hrsg.), Sprech- und Gesprächsstile, S. 94-122. Berlin: de Gruyter.

Günthner, Susanne und Hubert Knoblauch (1997): Forms are the food of faith - Gattungen als Muster kommunikativen Handelns. In: Kölner Zeitschrift für Soziologie und Sozialpsychologie, 46(4):693-723.

Hanke, Michael (2001): Kommunikation und Erzählung. Zur narrativen Vergemeinschaftungspraxis am Beispiel konversationellen Traumerzählens. Würzburg: Königshausen \& Neumann.

Hausendorf, Heiko (2012): Wie erzählt man einen Traum? Fragmente einer Ethnomethodologie der Traumkonversation. In: Ayaß, Ruth und Christian Meyer (Hrsg.), Sozialität in Slow Motion: Theoretische und empirische Perspektiven. Festschrift für Jörg Bergmann, S. 643-660. Wiesbaden: Springer VS.

Jefferson, Gail (1988): On the sequential organization of troubles-talk in ordinary conversation. In: Social Problems, 35(4):418-441.

Kächele, Horst, Cornelia Albani, Anna Buchheim, Michael Hölzer, Roderich Hohage, Juan Pablo Jimenez, Marianne Leuzinger-Bohleber, Erhard Mergenthaler, Lisbeth Neudert-Dreyer und Helmut Thomä (2006): The German specimen case Amalia X - Empirical studies. In: International Journal of Psychoanalysis, 87:809-826.

Knoblauch, Hubert (i. d. B.): Von kommunikativen Gattungen zu kommunikativen Formen: Konsequenzen des kommunikativen Konstruktivismus. In: Weidner, Beate et al. (Hrsg.), Verfestigungen in der Interaktion. Konstruktionen, sequenzielle Muster, kommunikative Gattungen. Berlin: de Gruyter.

Kotthoff, Helga (i. d. B.): Zum Indizieren schulorientierter Mutterschaft in Lehrperson-ElternGesprächen. In: Weidner, Beate et al. (Hrsg.), Verfestigungen in der Interaktion. Konstruktionen, sequenzielle Muster, kommunikative Gattungen. Berlin: de Gruyter.

Luckmann, Thomas (1986): Grundformen der gesellschaftlichen Vermittlung des Wissens Kommunikative Gattungen. In: Neidhardt, Friedhelm, Rainer M. Lepsius und Johannes Weiss (Hrsg.), Kultur und Gesellschaft (= Sonderheft 27 der Kölner Zeitschrift für Soziologie und Sozialpsychologie), S. 191-211. Opladen: Westdeutscher Verlag. 
Peräkylä, Anssi (2008): Psychoanalysis and conversation analysis: interpretation, affect and intersubjectivity. In: Peräkylä, Anssi et al. (Hrsg.), Conversation Analysis and Psychotherapy, S. 100-119. Cambridge: CUP.

Sacks, Harvey (1992 [1968]): Lecture 1: Second stories; “Mm hm;” Story prefaces; 'Local news;' Tellability. In: Jefferson, Gail (Hrsg.), Harvey Sacks: Lectures on Conversation, Vol.2, S. 3-16. Oxford: Blackwell.

Schütz, Alfred (1971): Gesammelte Aufsätze, Bd.1. Das Problem der sozialen Wirklichkeit: Kapitel Über die mannigfaltigen Wirklichkeiten, S. 237-298. Den Haag: Nijhoff.

Selting, Margret et al. (2009): Gesprächsanalytisches Transkriptionssystem 2 (GAT 2). In: Gesprächsforschung: Online-Zeitschrift zur verbalen Interaktion, 10:353-402.

Strümpell, Ludwig (1874): Die Natur und Entstehung der Träume. Leipzig: von Veit.

Tedlock, Barbara (1991): The new anthropology of dreaming. In: Dreaming, 1(2):161-178.

Zakharine, Dmitri (2005): Von Angesicht zu Angesicht. Der Wandel direkter Kommunikation in der ost- und westeuropäischen Neuzeit. Konstanz: UVK.

Zymner, Rüdiger (2010): Handbuch Gattungstheorie. Stuttgart: J. B. Metzler. 
